\title{
Research Trends of Acupuncture Therapy on Knee Osteoarthritis from 2010 to 2019: A Bibliometric Analysis
}

This article was published in the following Dove Press journal: Journal of Pain Research

\author{
Rongrong $\mathrm{Li} \mathbb{D}^{1, *}$ \\ Jing Sun ${ }^{1,2, *}$ \\ Hantong $\mathrm{Hu}^{1,2}$ \\ Qifei Zhang' \\ Ruohan Sun' \\ Shuning Zhou' \\ Haijuan Zhang (D) \\ Jianqiao Fang ${ }^{1,2}$
}

'The Third Clinical Medical College of Zhejiang Chinese Medical University, Hangzhou City, Zhejiang Province,

People's Republic of China; ${ }^{2}$ The Third Affiliated Hospital of Zhejiang Chinese Medical University, Hangzhou City, Zhejiang Province, People's Republic of China

*These authors contributed equally to this work
Correspondence: Jianqiao Fang Email fangjianqiao7532@163.com
Background: Acupuncture has been widely applied to relieve knee osteoarthritis (KOA) in many countries. However, the bibliometric analysis of the global use of acupuncture on KOA is largely unknown. Therefore, this study aimed to explore the current status and trends of the global use of acupuncture on KOA in recent 10 years by using CiteSpace (5.6.R3).

Methods: Publications regarding acupuncture therapy for treating KOA between 2010 and 2019 were extracted from the Web of Science database. CiteSpace was used to analyze the number of publications, countries, institutions, journals, authors, cited references and keywords by using standard bibliometric indicators.

Results: A total of 343 publications were retrieved from 2010 to 2019. The total number of publications continually increased over the past four years, and the most active journals, countries, institutions and authors in the field of acupuncture therapy on patients with KOA were identified. The Evid Based Complement Alternat Med (28) was the most prolific journal, and the Ann Intern Med (202) was the most cited journal. The most productive country and institution in this field was China (115) and University of York (18), respectively. Hugh Macpherson (18) was the most prolific author and Witt $\mathrm{C}$ ranked the first in the cited authors. In the ranking of frequency in cited reference, the first article was published by Scharf HP (54). The keyword of 'randomized controlled trial' ranked first for research developments with the highest citation burst (3.9486). Besides, there were three main frontiers in keywords for KOA research, including 'research method, 'age' and 'measure of intervention'.

Conclusion: The findings from this bibliometric study provide current status and trends in clinical research of acupuncture therapy on patients with KOA over the past ten years, which may help researchers identify hot topics and explore new directions for future research in this field.

Keywords: acupuncture, knee osteoarthritis, bibliometric analysis, CiteSpace

\section{Introduction}

Knee osteoarthritis (KOA) is one of the most common chronic degenerative diseases, which was characterized by joint pain, numbness, ankylosis and dysfunction, sometimes with progressive intraarticular cartilage and subchondral bone injury, synovitis, osteophyte formation, and joint cavity reduction. ${ }^{1,2}$ The genetics, age, female gender, obesity, overuse, misalignment and previous knee trauma are generally considered as high-risk factors for $\mathrm{KOA}^{3-6}$ About $22 \%$ of the general population suffers from KOA-associated pain, and the prevalence is higher in middle-age and elderly people. ${ }^{7}$ This disease constantly affected the quality of 
patients' life and caused some emotional disturbances, such as anxiety, depression and insomnia. ${ }^{8,9}$

Recently, there is no consensus on the best treatment to improve the symptoms of KOA. Standard pharmacological treatments always choose analgesics and nonsteroidal antiinflammatory drugs (NSAIDs) to alleviate symptoms in patients with KOA. However, it usually brings obvious adverse effects, such as gastrointestinal reactions, hepatorenal toxicity, and adverse events with the risk of cardiovascular. ${ }^{10-12}$ In recent years, exercise therapy was strongly recommended intervention for KOA based on international guideline, and most scholars advocated exercise therapy as the preferred rehabilitation method for KOA. ${ }^{13-}$ 15 Meanwhile, numbers of empirical studies have also demonstrated that exercise therapy could improve the quality of patients' life, effectively relieve the pain and promote functional recovery. ${ }^{16-18}$ However, poor long-term adherence is a serious problem that affects the therapeutic effect of exercise treatment. ${ }^{19}$ Apart from the aforementioned therapies, as a complementary therapy, acupuncture has become a favorable option for KOA treatment. Moreover, the efficacy of acupuncture on KOA has been confirmed by numerous randomized controlled trials, ${ }^{20-22}$ but the treatment method of KOA is still a research topic.

Bibliometrics refers to the cross-science of quantitative analysis of all knowledge carriers by using mathematical and statistical methods, and it is a statistical analysis and quantitative tool to study publications. ${ }^{23}$ Over the past ten years, studies have confirmed the effectiveness of acupuncture for KOA. ${ }^{24-26}$ However, to the best of our knowledge, a specific bibliometric analysis of the global use of acupuncture for KOA has not yet been performed. Therefore, it is essential to understand the current status of acupuncture research on KOA as a whole. This study aims to evaluate the research trends of acupuncture treatment on KOA and conduct a macroscopic overview of a significant amount of academic literature in the past ten years through bibliometric analysis.

CiteSpace is a visualization tool invented by Professor Chaomei Chen, which is characterized by co-occurrence network maps of countries, institutions, authors, keywords and co-citation network of cited journals, cited authors, cited references. ${ }^{27-29}$ In this study, CiteSpace was used to make a bibliometric analysis to explore the current status and research trends on the global use of acupuncture therapy on KOA within the past 10 years (from 2010 to 2019), and all eligible literature was extracted from the Web of Science database.
Table I The Topic Search Query

\begin{tabular}{|l|l|l|}
\hline Set & Results & Search Query \\
\hline$\# I$ & 28,361 & $\begin{array}{l}\text { (TS=((Osteoarthritis, Knee) OR (Knee } \\
\text { Osteoarthritis) OR (Knees Osteoarthritis) OR } \\
\text { (Osteoarthritis, Knees) OR (Osteoarthritis of } \\
\text { knee) OR (Osteoarthritis Of Knees) OR (Knee, } \\
\text { Osteoarthritis Of) OR (Knees, Osteoarthritis Of) } \\
\text { Indexes=Web of Science, Timespan=20I02019 }\end{array}$ \\
\hline$\# 2$ & 10,322 & $\begin{array}{l}\text { (TS=((Acupuncture Therapy)OR (Acupuncture } \\
\text { Treatments)OR (Acupuncture Treatment)OR } \\
\text { (Acupuncture)OR (body acupuncture)OR (Needle } \\
\text { Acupuncture)OR (Manual Acupuncture)OR } \\
\text { (Acupuncture Points)OR (Electroacupuncture)OR } \\
\text { (Warm Acupuncture)OR electro-acupuncture) } \\
\text { Indexes=Web of Science, Timespan=20I02019 }\end{array}$ \\
\hline$\# 3$ & 343 & \begin{tabular}{l} 
\#I AND \#2 \\
\hline
\end{tabular} \\
\hline
\end{tabular}

\section{Methods}

All data in this study were obtained from the Web of Science on 1 March 2020. The data search strategy included the topic "knee osteoarthritis" and "acupuncture therapy", with the publications period of the literature ranging from 2010 to 2019. In addition, the countries, categories and language of publications were not been restricted. The specific search strategies and results are shown in Table 1.

Bibliometric analysis was performed to analyze the trends and patterns in the identified publications by using CiteSpace (5.6.R3) software, which was used to clarify the annual output counts, the prolific journals, authors,

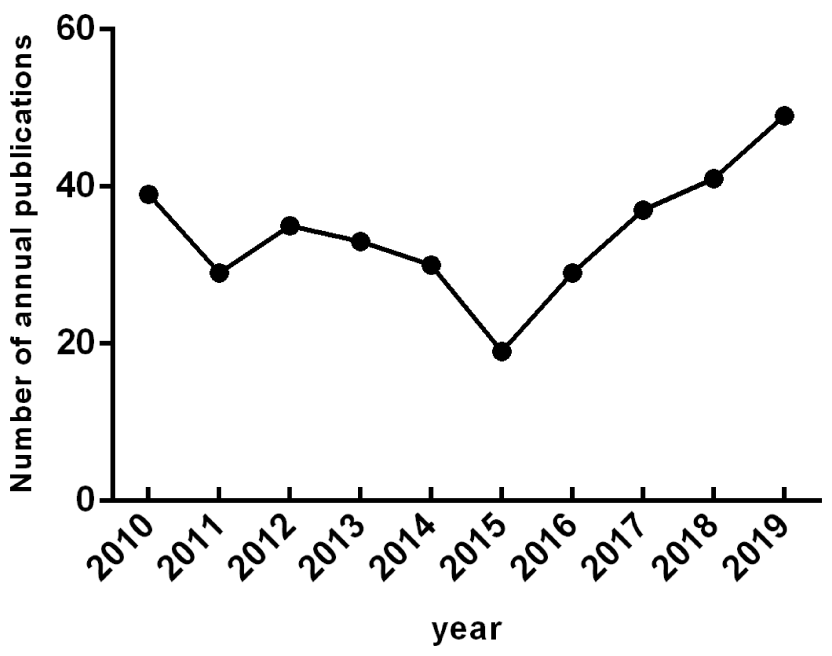

Figure I The annual number of publications on acupuncture treatment for KOA between 2010 to 2019 . 
Table 2 Top 10 Scholarly Journals Related to Acupuncture Therapy on KOA

\begin{tabular}{|c|c|c|c|c|c|c|c|}
\hline Rank & Publications & Journal & $\begin{array}{l}\text { IF } \\
(2019)\end{array}$ & Rank & Publications & Journal & $\begin{array}{l}\text { IF } \\
(2019)\end{array}$ \\
\hline I & 28 & $\begin{array}{l}\text { Evidence-Based Complementary } \\
\text { And Alternative Medicine }\end{array}$ & 1.984 & 6 & 9 & $\begin{array}{l}\text { Journal of Alternative And } \\
\text { Complementary Medicine }\end{array}$ & 1.868 \\
\hline 2 & 24 & Acupuncture In Medicine & 2.637 & 7 & 8 & BMJ Open & 2.376 \\
\hline 3 & 17 & Trials & 1.975 & 8 & 8 & $\begin{array}{l}\text { Journal of Traditional Chinese } \\
\text { Medicine }\end{array}$ & 0.907 \\
\hline 4 & 14 & Osteoarthritis And Cartilage & 4.879 & 9 & 8 & Plos One & 2.776 \\
\hline 5 & 9 & $\begin{array}{l}\text { BMC Complementary and } \\
\text { Alternative Medicine }\end{array}$ & 2.479 & 10 & 7 & $\begin{array}{l}\text { Complementary Therapies In } \\
\text { Medicine }\end{array}$ & 1.979 \\
\hline
\end{tabular}

institutions and countries. $^{29}$ Meanwhile, we also researched the cooperation relationships, such as cooccurrence analysis of keywords, authors, references and institutions. The current research basis, cutting-edge knowledge and research trends of acupuncture treatment on KOA were obtained by bibliometric visualized analysis.

The parameters of CiteSpace were set as below: time slicing was from 2010 to 2019, years per slice (1), all options in the term source were selected, one node type was selected at a time, selection criteria (top 50 objects), and pruning (pathfinder). Visualization knowledge figures were mainly composed of nodes and links. Each node in the map represented an element, such as author, keyword, institution, etc. The size of node, which commonly suggested the frequency of appearance or citation, and different colors of nodes indicated the different years and the circles of different colors represented the year of 2010 to 2019 from the inside to the outside of the nodes. Furthermore, lines between the nodes signified cooperation or co-occurrence or co-citation relationships. The purple circle represented centrality, nodes with high centrality were usually considered as pivotal points or key points in a specific field. ${ }^{30,31}$

\section{Results and Discussion}

\section{Annual Publications}

By searching for the topic words in Web of Science, a total of 343 articles were included. The number of specific articles published each year is shown in Figure 1. In our study, we found that the number of publications on acupuncture therapy of KOA has fluctuated over the past 10 years. From 2010 to 2011, the number of publications decreased from 39 references to 29 references. Although the number of publications increased again in 2012, it did not restore the level of 2010. From 2012 to 2015, the number of published articles decreased significantly, and the lowest publications were in 2015, with only 19 publications. However, the number of publications increased rapidly in 2016. Furthermore, the number of publications increased continually from 2015 to 2019. The trend of publications' number indicated that acupuncture, as a complementary treatment, has received more attention, and more studies have been carried out to observe the efficacy of acupuncture on KOA in recent years.

\section{Analysis of Journals and Cited Journals}

The top 10 journals of acupuncture treatment on KOA are shown in Table 2. The average impact factor (IF) of the top 10 journals was 2.386, and Evidence-Based Complementary And

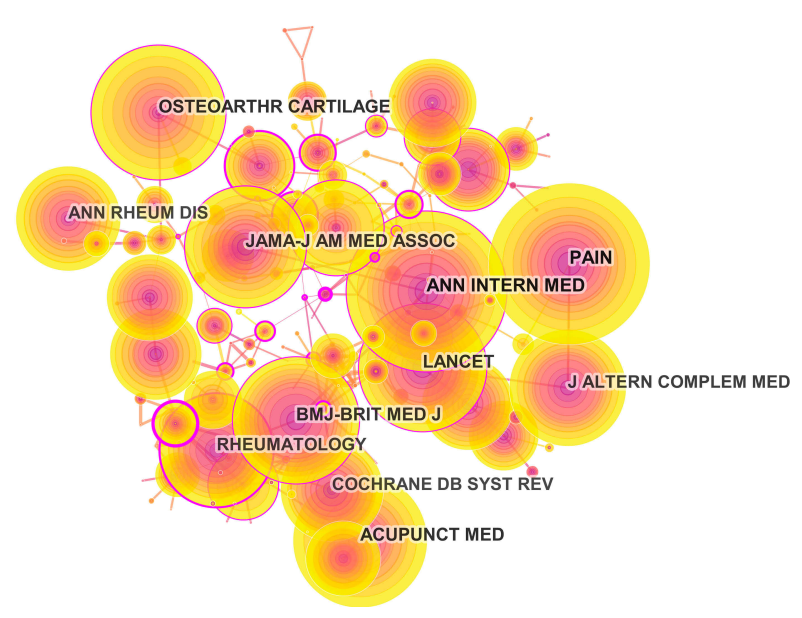

Figure 2 Cited journal maps related to acupuncture treatment for KOA research from 2010 to 2019. The nodes in the map represent journals, and lines between the nodes represent co-citation relationships. The various colors in the nodes represent the different years. The larger the node area, the greater the number of cocitations. The purple ring represents centrality, and nodes with high centrality are considered as pivotal points in the literature. 
Table 3 Top 5 Cited Journals and Centrality Related to Acupuncture Therapy on KOA

\begin{tabular}{|l|l|l|l|l|l|}
\hline Rank & Cited Journal & Frequency & Rank & Cited Journal & Centrality \\
\hline 1 & Ann Intern Med & 202 & 1 & Trials & 0.47 \\
2 & Pain & 201 & 2 & Anesth. Analg & 0.43 \\
3 & Osteoaryhr Cartilage & 168 & 3 & Clin Rheumatol & 0.37 \\
4 & Acupunct Med & 167 & 4 & JCR-J Clin Rheumatol & 0.37 \\
5 & Lancet & 160 & 5 & Clin Rehabil & 0.34 \\
\hline
\end{tabular}

Alternative Medicine was the most productive journal, with a total of 28 articles, which was followed by Acupuncture In Medicine, with a total of 24 articles. Furthermore, we found that the Osteoarthritis And Cartilage with the highest IF is a professional journal in this field, with an IF of 4.879. The cocitation combined with centrality, a cited journal map was generated by CiteSpace, including the total of 5773 references (shown in Figure 2 and Table 3). The nodes in the map represented journals, and links between the nodes meant cocitation relationships. In addition, the purple ring indicated the centrality of literature, it was crucial that nodes with high centrality were usually considered as pivotal points in the literature. Thus, the first in the frequency and centrality were Ann Intern Med, and Trails, respectively. In the journal cited in 202 records of Ann Intern Med, one article got the larger citation, which performed a meta-analysis of acupuncture therapy on 4 chronic pain diseases, and manifested the efficacy of acupuncture was better than both of no-acupuncture control and sham acupuncture in the treatment of KOA. Meanwhile, the results from the meta-analyses provided that the most robust evidence to date that acupuncture is a reasonable referral option for patients with $\mathrm{KOA}^{32}$

\section{Distribution of Countries and Institutions}

Distribution of countries map was generated, 16 nodes and 53 links composed of the merged network (Figure 3). The 343 references were published by researchers in 16 countries, and the top 5 countries for centrality (purple ring) were England (1.28), Canada (0.97), Sweden (0.63), USA (0.46), and Denmark (0.46). Besides, the top 10 countries of publications are displayed in Table 4. A total of 115 references were published in China, which may be related to the fact that acupuncture originated from China. USA was ranked in the second position (109), thereby, indicating that acupuncture treatment for KOA has been widely used in the USA. Additionally, China and South Korea were the most productive countries in Asia, while the USA and England were the most prolific countries in the West (Table 4). Further analysis revealed that more high-quality randomized controlled trails of acupuncture therapy on KOA were being conducted. ${ }^{33-36}$

Distribution of institutions map consists of 62 nodes and 106 links (Figure 4). A total of 62 institutions were dedicated to the research of acupuncture treatment on KOA, among which the top 5 institutions of publications were University of York (18), Beijing University of Chinese Medicine (13), Chengdu University of Traditional Chinese Medicine (12), University of Maryland (9), and University of Southampton (9), respectively (Table 5). Interestingly, University of York was not only the most prolific institution, but also the highest centrality institution, suggesting that University of York is the most important institution for researching acupuncture treatment of KOA. Meanwhile, the top 3 centrality institutions included University of York, University of Maryland and Chinese Academy of Medical Sciences. Therefore, based on the publications and centrality, we found that the institutions from England, USA, and China paid more concerns on the research of acupuncture treatment in KOA currently, and the University of York, Sichuan University and University of Southampton were the strongest cooperation institutions.

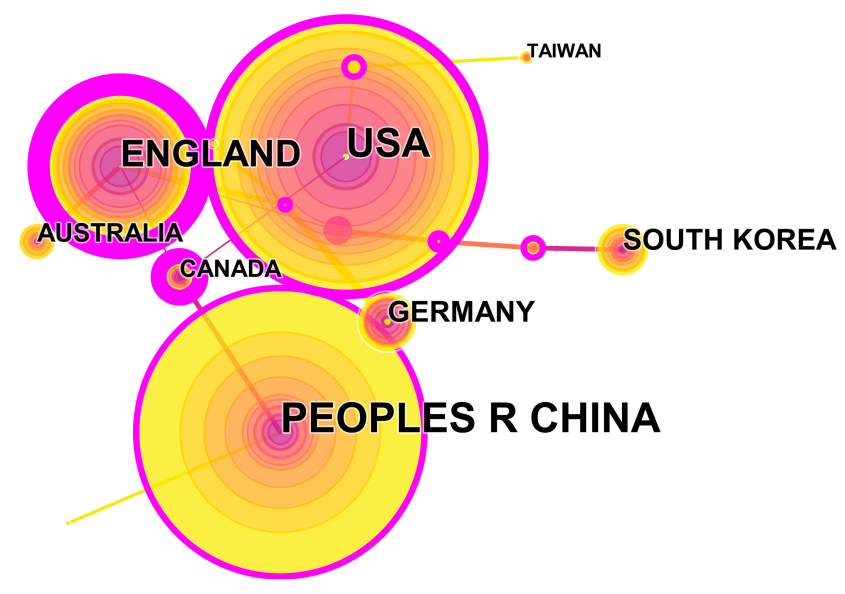

Figure 3 Map of countries researching acupuncture for KOA from 2010 to 2019 . The nodes in the map represent countries or territories. The lines between the nodes represent cooperation relationships. The various colors in the nodes represent the different years, and the larger the area of the node, the larger the number of publications. The purple ring represents centrality, and nodes with high centrality are considered as pivotal points in the literature. 
Table 4 Top 10 Publications and Centrality of Countries Related to Acupuncture Therapy on KOA

\begin{tabular}{|l|l|l|l|l|l|}
\hline Rank & Publications & Countries & Rank & Centrality & Countries \\
\hline 1 & 115 & Peoples R China & 1 & 1.28 & England \\
2 & 109 & USA & 2 & 0.97 & Canada \\
3 & 61 & England & 3 & 0.63 & Sweden \\
4 & 27 & Germany & 4 & 0.46 & USA \\
5 & 24 & South Korea & 5 & 0.46 & Denmark \\
6 & 17 & Australia & 6 & 0.25 & Peoples R China \\
7 & 13 & Canada & 7 & 0.25 & Netherlands \\
8 & 8 & Taiwan & 8 & 0.25 & Brazil \\
9 & 7 & Netherlands & 9 & 0.25 & Switzerland \\
10 & 6 & Brazil & 10 & 0 & Germany \\
\hline
\end{tabular}

\section{Analysis of Authors and Cited Authors}

The authors of the 343 publications were analyzed and resulted in 104 nodes and 303 links (Figure 5), indicating that the 343 articles were published by 104 authors. The map of authors was designed to reveal the most prolific author or co-author, as well as demonstrate the closeness of collaboration among the authors, which could provide information on influential research groups and potential collaborators, and help researchers establish cooperative relationships. The top
5 authors were Hugh Macpherson, Claudia M Witt, Lixing LAO, Andrew J Vickers, and Adrian White (Table 6). Among them, the most prolific author was Hugh Macpherson, from the University of York in the United Kingdom, with 13 articles. The cost-effectiveness study of assisted non-pharmacological interventions for KOA performed by Hugh Macpherson found that high-quality KOA clinical trials were urgently needed and the effective cost of acupuncture was the lowest among non-pharmacological

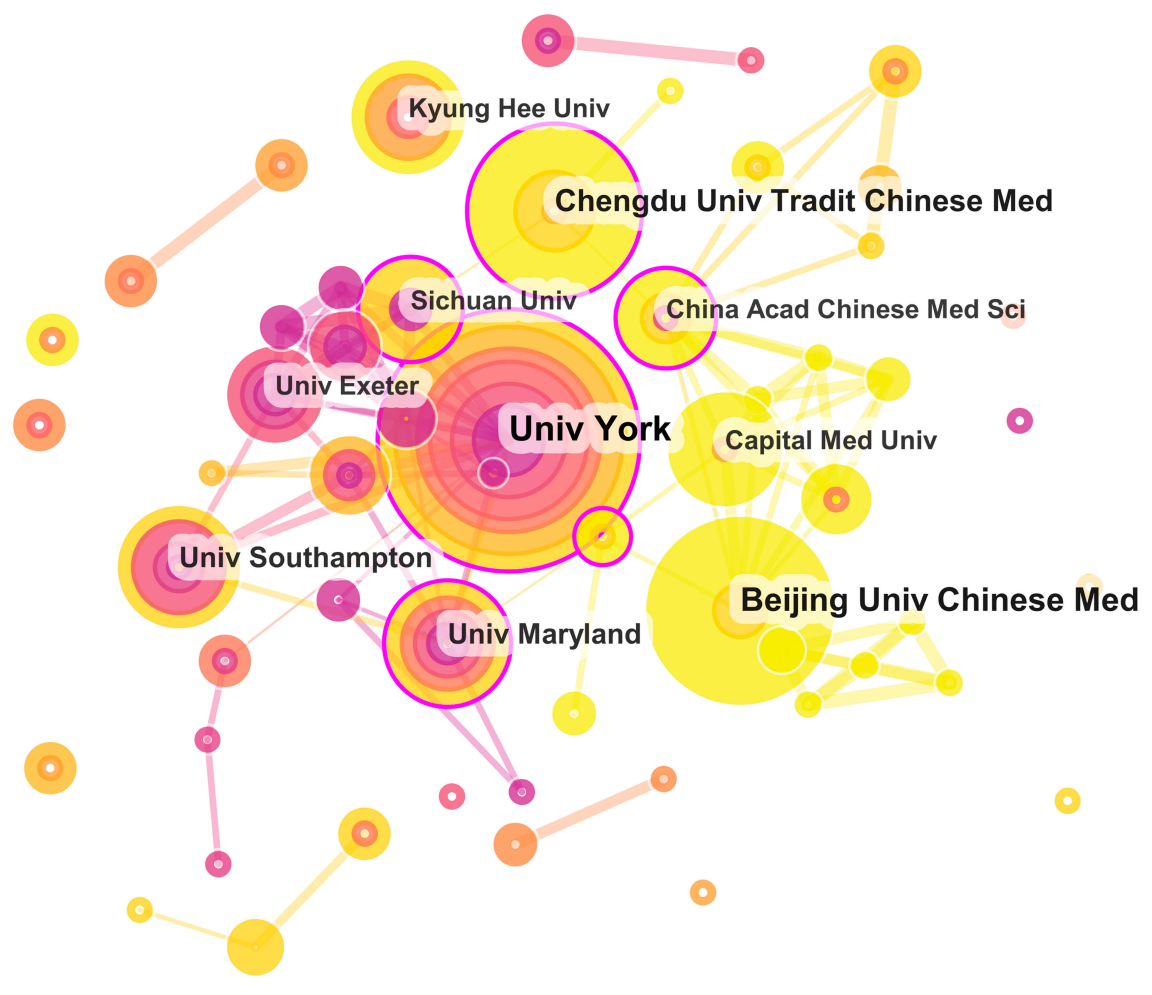

Figure 4 Map of institutions researching acupuncture for KOA from 2010 to 2019 . The nodes in the map represent institutions, and lines between the nodes represent collaborative relationships. The various colors in the nodes represent the different years, and the larger the node area, the larger the number of publications. The purple ring represents centrality, and nodes with high centrality are considered as pivotal points in the literature. 
Table 5 Top 10 Publications and Centrality of Institutions Related to Acupuncture Therapy on KOA

\begin{tabular}{|l|l|l|l|l|l|}
\hline Rank & Publications & Institutions & Rank & Centrality & Institutions \\
\hline 1 & 18 & Univ York & 1 & 0.29 & Univ York \\
2 & 13 & Beijing Univ Chinese Med & 2 & 0.27 & Univ Maryland \\
3 & 12 & Chengdu Univ Tradit Chinese Med & 3 & 0.24 & Univ Hong Kong \\
4 & 9 & Univ Maryland & 4 & 0.21 & Capital Med Univ \\
5 & 9 & Univ Southampton & 5 & 0.09 & Sichuan Univ \\
6 & 8 & Capital Med Univ & 6 & 0.09 & China Acad Chinese Med Sci \\
7 & 8 & Kyung Hee Univ & 7 & 0.08 & Beijing Hosp Tradit Chinese \& Western Med \\
8 & 7 & China Acad Chinese Med Sci & 8 & 0.08 & Hebei Univ Chinese Med \\
9 & 7 & Sichuan Univ & 9 & 0.06 & Charite \\
10 & 7 & Univ Exeter & 10 & 0.03 & Keele Univ \\
\hline
\end{tabular}

interventions. ${ }^{37}$ In addition, some of his system reviews and meta-analysis articles also indicated that referral acupuncture treatment was a reasonable choice for patients with KOA. ${ }^{38,39}$ However, there was still a lack of high-quality randomized controlled trials to provide sufficient evidence to demonstrate the therapeutic effects of acupuncture therapy on KOA. Moreover, the links showed that the cooperation between authors in this field was not close, suggesting that authors can collaborate to produce more high-quality articles in the future.

The map of cited authors was composed of 408 nodes and 1348 links (Figure 6). Witt $\mathrm{C}$ had the highest citation counts (102), followed by Berman BM (99), Manheimer E (97), Scharf HP (84) and Bellamy N (72) (Table 7). The top 5 centrality of cited authors included CHEN LX (0.31), Barnes PM (0.26), White A (0.25), Bennell KL (0.25) and
Diener HC (0.19) (Table 7). A comprehensive analysis found that Witt C, Berman BM, CHEN LX and Barnes PM were the professors in the field, and had an important influence on the development of acupuncture treatment of KOA. Witt $\mathrm{C}$ ranked the first in the cited author frequency and he is a professor at Charite University Medical Center. One of her articles pointed out that the results of randomized controlled trials were summarized in systematic reviews and meta-analysis, these reviews and meta-analysis were often used to provide decision-makers with decision-making information. However, it was necessary to carry out relevant researches to obtain more evidences about the effectiveness and cost-effectiveness of acupuncture therapy on KOA. In addition, a further understanding of the mechanism of acupuncture therapy on KOA was also urgently warranted. ${ }^{40}$ Berman BM, who is from the University of Maryland

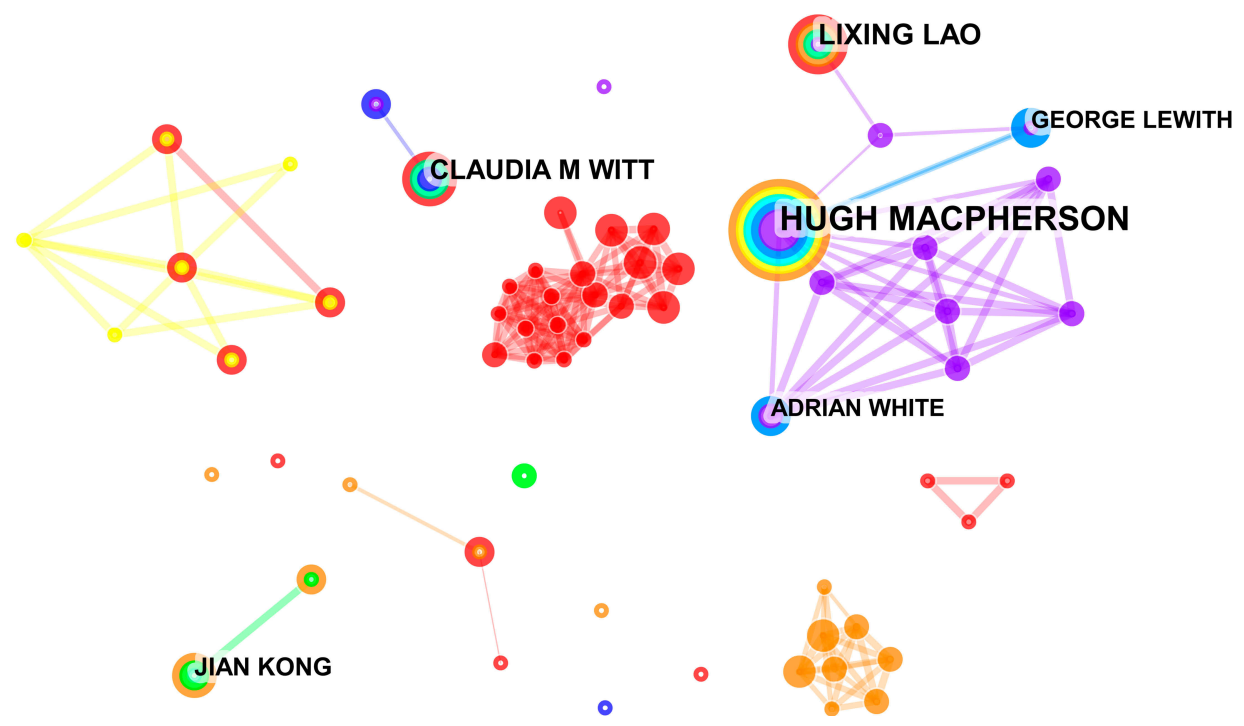

Figure 5 Map of author related to acupuncture for KOA from 2010 to 2019 . The nodes in the map represent authors, and lines between the nodes represent the collaborative relationships. The various colors in the nodes represent the different years, and the larger the node area, the greater the number of publications. 
Table 6 Top 10 Prolific Authors Related to Acupuncture Therapy on KOA

\begin{tabular}{|l|l|l|l|l|l|}
\hline Rank & Publication & Author & Rank & Publication & Author \\
\hline 1 & 13 & Hugh Macpherson & 6 & 6 & Jian KONG \\
2 & 8 & Claudia M Witt & 7 & 5 & Cunzhi LIU \\
3 & 8 & Lixing LAO & 8 & 5 & George Lewith \\
4 & 7 & Andrew J Vickers & 9 & 4 & Yong TANG \\
5 & 6 & Adrian White & 10 & 4 & Lize XIONG \\
\hline
\end{tabular}

School of Medicine, mainly devoted to investigate the mechanism of pain, one of his opinion was that acupuncture treatment provided more significant improvement in function and pain relief as an adjunctive therapy on KOA when compared with credible sham acupuncture and education control groups. Hence, acupuncture may have an important role to relieve symptoms related to $\mathrm{KOA}^{41}$

\section{Analysis of Cited References}

The top 5 frequency and centrality of cited references were shown in Tables 8 and 9. By analyzing the literature with high co-citation frequency and centrality, the knowledge foundation of the subject could be obtained. The top 3 articles were all the randomized controlled trials of acupuncture treatment on KOA and were published in Ann Intern Med, Lancet and JAMA, respectively, ${ }^{42-44}$ indicating that the importance of these articles in this field, which also had a greater influence in this field. Additionally, the

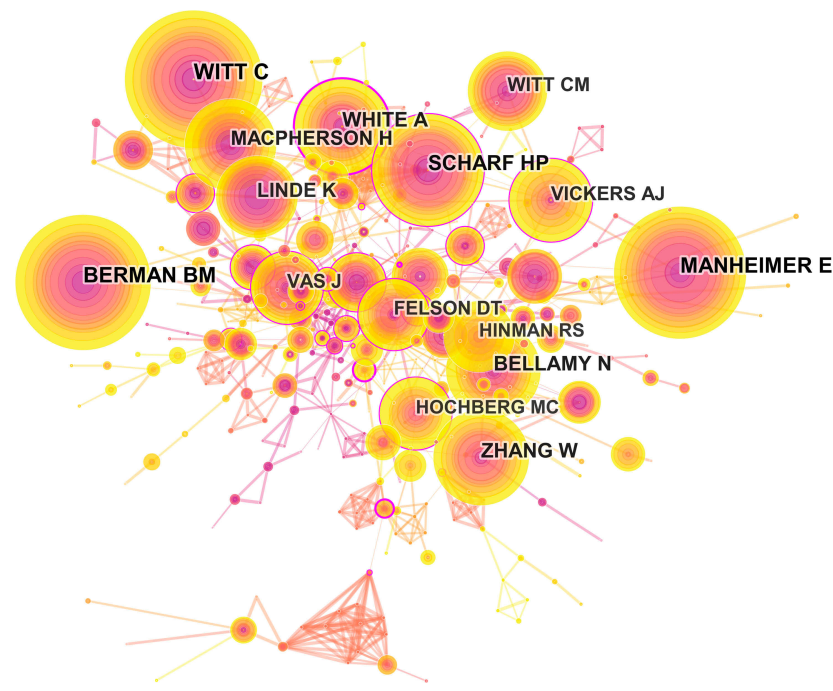

Figure 6 Map of cited author related to acupuncture for KOA from 2010 to 2019. The nodes in the map represent co-cited authors, and lines between the nodes represent co-citation relationships. The various colors in the nodes represent the different years. The larger the node area, the greater the number of co-citations. The purple ring represents centrality, and nodes with high centrality are considered to be pivotal points in the literature. meta-analysis published by Vickers AJ, which was ranked the fourth list, suggested that acupuncture was an effective method for treating chronic osteoarthritis pain, the therapeutic effect of acupuncture persisted with the progress of time and could not be explained by the placebo effects only. ${ }^{32}$ The centrality of cite references ranked the first conducted by Mavrommatis CI, who carried out a 3-armed, single-blind, randomized, sham-controlled trail to compare the efficacy of acupuncture and medicine in the treatment of KOA, and results revealed that acupuncture combined with medicine had the best curative effect. $^{45}$ A total of 9372 records were generated by 343 publications to analysis cited references, and the map with 538 nodes and 1880 links were obtained (Figure 7). Over the past decade, the comprehensive analysis mainly focused on randomized controlled trials of acupuncture treatment for KOA, individual patient data (IPD) metaanalysis, analgesic mechanisms based on nerves and receptors, researching placebo effects, and developing the corresponding guidelines for the treatment of KOA.

\section{Analysis of Keywords}

It was considered that the indicators for evaluating the most cutting-edge themes or emerging trends were the increased number of keyword burst in citation or the increased frequency of keywords within a certain period of time. ${ }^{46}$ The map of keywords co-occurrence was generated and consisted of 160 nodes and 616 links (Figure 8). According to the frequency and centrality, we found the popular keywords were "acupuncture", "osteoarthriti", "pain", "knee”, "knee osteoarthriti", "randomized controlled trial", "intervention" and "meta-analysis" (Table 10). The top 15 keywords with the strongest citation burst from 2010 to 2019 are shown in Figure 9. The most recent burst keywords were "randomized controlled trial", "double blind", "older adult", "OARSI recommendation", "placebo", "expectation", "electroacupuncture (EA)", "safety" and "alternative medicine". The 3 frontiers in burst strength were "research method, "age" and "measure of intervention". Randomized controlled trial 
Table 7 Top 5 Frequency and Centrality of Cited Authors Related to Acupuncture Therapy on KOA

\begin{tabular}{|l|l|l|l|l|l|}
\hline Rank & Frequency & Author & Rank & Centrality & Author \\
\hline 1 & 102 & Witt C & 1 & 0.31 & CHEN LX \\
2 & 99 & Berman BM & 2 & 0.26 & Barnes PM \\
3 & 97 & Manheimer E & 3 & 0.25 & White A \\
4 & 84 & Scharf HP & 4 & 0.25 & Bennell KL \\
5 & 72 & Bellamy N & 5 & 0.19 & Diener HC \\
\hline
\end{tabular}

was the most important research method to evaluate the efficacy of acupuncture therapy on KOA, which could also provide high-quality evidence-based testimony. Meanwhile, it was necessary to declare the implementation of blind method. ${ }^{47}$ However, it was difficult to achieve double blinding for operators and patients in acupuncture studies. Hence, some single-blind trials had been conducted. ${ }^{45,48}$ Data have shown that more than $19.4 \%$ of people over the age of 60 years suffered from KOA, which seriously affected their quality of life. Besides, more than 20 million Americans, as well as 35 to 40 million Europeans experienced KOA, especially in elderly individuals. ${ }^{49,50}$ Therefore, this phenomenon highlights the importance of studying KOA in the older adults. The keyword "electroacupuncture", emerging from

Table 8 Top 5 Frequency of Cited References Related to Acupuncture Therapy on KOA

\begin{tabular}{|l|l|l|l|}
\hline Rank & Frequency & References & $\begin{array}{l}\text { Author and } \\
\text { Publication } \\
\text { Year }\end{array}$ \\
\hline 1 & 54 & $\begin{array}{l}\text { Acupuncture and knee } \\
\text { osteoarthritis: a three- } \\
\text { armed randomized trial }\end{array}$ & $\begin{array}{l}\text { Scharf HP } \\
(2006)\end{array}$ \\
\hline 2 & 46 & $\begin{array}{l}\text { Acupuncture in patients } \\
\text { with osteoarthritis of the } \\
\text { knee: a randomised trial }\end{array}$ & $\begin{array}{l}\text { Witt } \\
\text { C (2005) }\end{array}$ \\
\hline 3 & 45 & $\begin{array}{l}\text { Acupuncture for chronic } \\
\text { knee pain: a randomized } \\
\text { clinical trial }\end{array}$ & $\begin{array}{l}\text { Hinman RS } \\
(2014)\end{array}$ \\
\hline 4 & 44 & $\begin{array}{l}\text { Acupuncture for chronic } \\
\text { pain: individual patient data } \\
\text { meta-analysis }\end{array}$ & $\begin{array}{l}\text { Vickers AJ } \\
(2012)\end{array}$ \\
\hline 5 & 38 & $\begin{array}{l}\text { American College of } \\
\text { Rheumatology 20I2 } \\
\text { recommendations for the } \\
\text { use of nonpharmacologic } \\
\text { and pharmacologic } \\
\text { therapies in osteoarthritis } \\
\text { of the hand, hip, and knee }^{46}\end{array}$ & $\begin{array}{l}\text { Hochberg } \\
\text { MC (20I2) }\end{array}$ \\
\hline
\end{tabular}

2018, has shown the strength of citation burst of 2.8472 , thereby indicating that EA had gained more popularity than manual acupuncture in recent years. A study was conducted to investigate the optimal intensity of EA for patients with $\mathrm{KOA}$, and the results showed that strong EA $(>2 \mathrm{~mA})$ was

Table 9 Top 5 Centrality of Cited References Related to Acupuncture Therapy on KOA

\begin{tabular}{|c|c|c|c|}
\hline Rank & Centrality & References & $\begin{array}{l}\text { Author and } \\
\text { Publication } \\
\text { Year }\end{array}$ \\
\hline I & 0.21 & $\begin{array}{l}\text { Acupuncture as an } \\
\text { adjunctive therapy to } \\
\text { pharmacological treatment } \\
\text { in patients with chronic pain } \\
\text { due to osteoarthritis of the } \\
\text { knee: a 3-armed, } \\
\text { randomized, placebo- } \\
\text { controlled trial }\end{array}$ & $\begin{array}{l}\text { Mavrommatis } \\
\mathrm{Cl}, 2012\end{array}$ \\
\hline 2 & 0.18 & $\begin{array}{l}\text { American College of } \\
\text { Rheumatology } 2012 \\
\text { recommendations for the } \\
\text { use of nonpharmacologic } \\
\text { and pharmacologic } \\
\text { therapies in osteoarthritis } \\
\text { of the hand, hip, and knee }\end{array}$ & $\begin{array}{l}\text { Hochberg MC } \\
(2012)\end{array}$ \\
\hline 3 & 0.15 & $\begin{array}{l}\text { A randomized trial } \\
\text { comparing acupuncture, } \\
\text { simulated acupuncture, and } \\
\text { usual care for chronic low } \\
\text { back pain }{ }^{47}\end{array}$ & $\begin{array}{l}\text { Cherkin DC, } \\
2009\end{array}$ \\
\hline 4 & 0.14 & $\begin{array}{l}\text { Convincing evidence from } \\
\text { controlled and uncontrolled } \\
\text { studies on the lipid- } \\
\text { lowering effect of a statin }{ }^{48}\end{array}$ & $\begin{array}{l}\text { Higgins J, } \\
2012\end{array}$ \\
\hline 5 & 0.13 & $\begin{array}{l}\text { The modulation effect of } \\
\text { longitudinal acupuncture on } \\
\text { resting state functional } \\
\text { connectivity in knee } \\
\text { osteoarthritis patients }\end{array}$ & $\begin{array}{l}\text { Chen XY, } \\
2015\end{array}$ \\
\hline
\end{tabular}




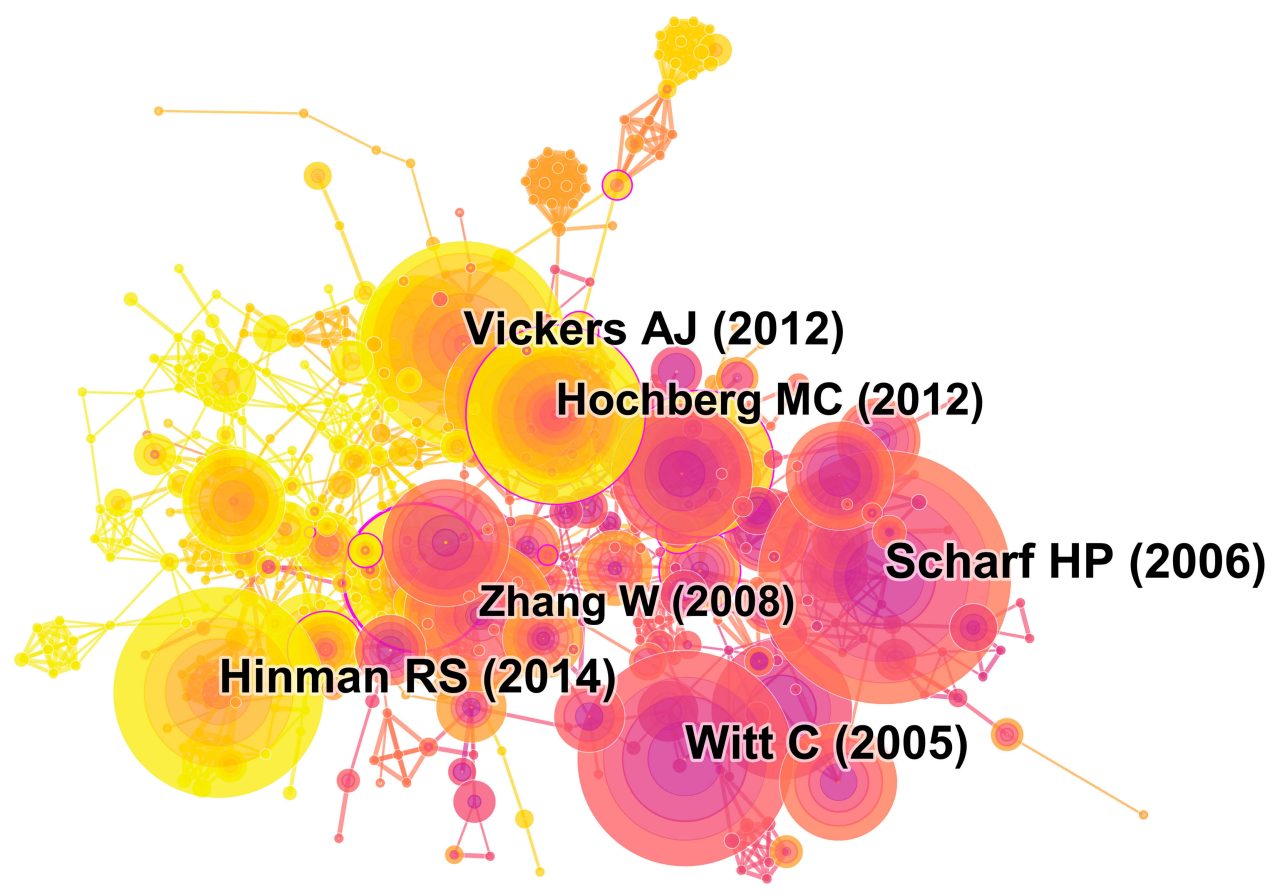

Figure 7 Map of cited references related to acupuncture for KOA from 2010 to 2019. The nodes in the map represent cited references, and lines between the nodes represent co-citation relationships. The various colors in the nodes represent the different years, and the larger the node area, the greater the number of co-citations. The purple ring represents centrality, and nodes with high centrality are considered as pivotal points in the literature.

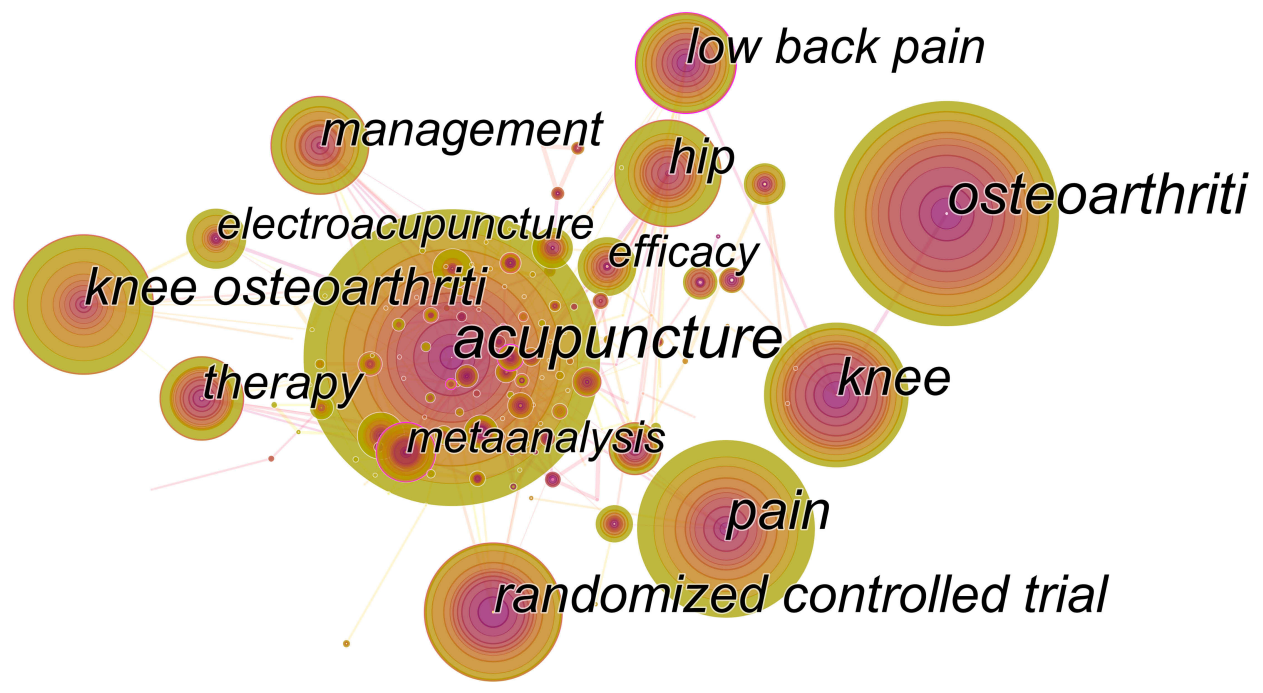

Figure 8 Map of keywords occurrence related to acupuncture for KOA from 2010 to 2019. The nodes in the map represent keywords. The lines between the nodes represent co-occurrence relationships. The various colors in the nodes represent the different years, and the larger the node area, the higher the frequency. The purple ring represents centrality, and nodes with high centrality are considered as pivotal points in the literature.

better than weak or sham EA in alleviating pain intensity and inhibiting chronic pain. ${ }^{35}$ However, studies found that the efficacy of EA on pain relief and function improvement in patients with KOA was also not confirmed when compared to manual acupuncture. ${ }^{25}$ Therefore, more studies should be carried out for further investigation.
Some limitations should be addressed in our study. Firstly, we only analyzed the documents recorded in the Web of Science. Therefore, our findings may not be comprehensive, and it is necessary to perform further analysis of literature in other databases, such as some Chinese databases. In addition, although the research topic had 
Table 10 Top 10 Frequency and Centrality of Keywords Related to Acupuncture Therapy on KOA

\begin{tabular}{|l|l|l|l|l|l|}
\hline Rank & Keyword & Frequency & Rank & Keyword & Centrality \\
\hline 1 & Acupuncture & 170 & 1 & Meta-analysis & 0.30 \\
2 & Osteoarthriti & 131 & 2 & Randomized controlled trial & 0.30 \\
3 & Pain & 105 & 3 & Low back pain & 0.24 \\
4 & Knee & 87 & 4 & Intervention & 0.22 \\
5 & Knee osteoarthriti & 84 & 5 & Trigger point & 0.20 \\
6 & Randomized controlled trial & 82 & 6 & Systematic review & 0.18 \\
7 & Hip & 66 & 7 & Neck pain & 0.17 \\
8 & Low back pain & 61 & 8 & Pharmacological treatment & 0.17 \\
9 & Management & 60 & 9 & Therapy & 0.16 \\
10 & Therapy & 53 & 10 & Primary care & 0.15 \\
\hline
\end{tabular}

been defined when searching, we cannot guarantee that every document is completely related to the topic. However, we believe that this study can still be used to describe the overall situation and trends in this field.

\section{Conclusion}

Acupuncture, as a complementary and alternative therapy, was widely used in patients with $\mathrm{KOA}$, and has the advantages of high safety and fewer side effects. In this study, a large number of publications were published in the journals of Evidence-Based Complementary And Alternative Medicine. The most prolific country was China. However, the most productive institution of publications in this field was the University of York in England, it may reveal the fact that acupuncture as a complementary and alternative therapy to treat patients with KOA was becoming increasingly accepted in the world. In addition, recent results indicated a lack of cooperation between different institutions and countries. Therefore, cooperation and connection between different research teams should be strengthened, which will be beneficial to promote the current opinion of acupuncture pain control in KOA as an alternative method. Moreover, randomized controlled trial is currently the main research method to study acupuncture treatment on KOA. Given that it is difficult

\begin{tabular}{cllll} 
Keywords & \multicolumn{5}{c}{ Year Strength Begin End } & $\mathbf{2 0 1 0}$ - 2019 \\
randomized controlled trial & 2010 & $\mathbf{3 . 9 4 8 6}$ & 2010 & 2011 \\
double blind & 2010 & $\mathbf{3 . 7 5 3 2}$ & 2014 & 2016 \\
older adult & 2010 & $\mathbf{3 . 4 7 9 7}$ & 2012 & 2013 \\
oarsi recommendation & 2010 & $\mathbf{3 . 1 6 6 2}$ & 2013 & 2015 \\
placebo & 2010 & $\mathbf{3 . 0 4 2 4}$ & 2010 & 2010 \\
expectation & 2010 & $\mathbf{3 . 0 2 6 3}$ & 2010 & 2011 \\
migraine & 2010 & $\mathbf{2 . 9 5 0 8}$ & 2010 & 2013 \\
electroacupuncture & 2010 & $\mathbf{2 . 8 4 7 2}$ & 2018 & 2019 \\
kneeosteoarthriti & 2010 & $\mathbf{2 . 5 6 2 3}$ & 2010 & 2012 \\
safety & 2010 & $\mathbf{2 . 4 7 0 1}$ & 2015 & 2017 \\
medicine & 2010 & $\mathbf{2 . 3 6 8 5}$ & 2010 & 2012 \\
alternative medicine & 2010 & $\mathbf{2 . 3 5 7 2}$ & 2010 & 2013 \\
complementary & 2010 & $\mathbf{2 . 3 3 8 7}$ & 2010 & 2013 \\
irritable bowel syndrome & 2010 & $\mathbf{2 . 3 1 5 6}$ & 2010 & 2010 \\
knee & 2010 & $\mathbf{2 . 1 9 2 3}$ & 2010 & 2011
\end{tabular}

Figure 9 Top 15 keywords with the strongest citation bursts. The red bars demonstrated that the keyword was cited frequently, the green bars showed that the keyword was cited infrequently. 
to achieve double blinding during acupuncture manipulation, single blind randomized controlled trials are frequently performed to verify the effect of acupuncture for KOA. Metaanalysis was the main methodology to evaluate the efficacy of acupuncture therapy on KOA, but the high-quality evaluation of various acupuncture treatments was inadequate currently, which is need further research by physiotherapists.

In summary, this study provides potential collaborators and institutions, and hot topics, thereby providing a perspective to the developing trend of acupuncture therapy on KOA, which may help researchers explore new directions for future research in this field.

\section{Data Sharing Statement}

The raw data can be directly obtained from the Web of Science Core Collection (WoSCC).

\section{Acknowledgments}

The authors would like to express their appreciation to Professor Chaomei Chen for inventing the CiteSpace and making it free to use.

\section{Funding}

This study was supported by the key plan of Zhejiang Province Traditional Chinese Medicine Prevention and Treatment of Major Disease of the Health and Family Planning Commission of Zhejiang Province (No. 2018ZY008). The trial sponsor is the Third Affiliated Hospital of Zhejiang Chinese Medicine University (219 Moganshan Road, Xihu District, Hangzhou City, Zhejiang Province 310005, China, 86-571-88393504).

\section{Authors' Contributions}

All authors made substantial contributions to conception and design, acquisition of data, or analysis and interpretation of data; took part in drafting the article or revising it critically for important intellectual content; gave final approval of the version to be published; and agree to be accountable for all aspects of the work.

\section{Disclosure}

The authors report no conflicts of interest in this work.

\section{References}

1. Zheng Y, Duan X, Qi S, et al. Acupuncture therapy plus hyaluronic acid injection for knee osteoarthritis: a meta-analysis of randomized controlled trials. Evid Based Complement Alternat Med. 2020;2020:4034105. doi:10.1155/2020/4034105
2. S H, SJ B. Yoga for arthritis: a scoping review. Rheum Dis Clin North Am. 2011;37(1):33-46. doi:10.1016/j.rdc.2010.11.001

3. Bijlsma JW, Berenbaum F, Lafeber FP. Osteoarthritis: an update with relevance for clinical practice. Lancet. 2011;377(9783):2115-2126. doi:10.1016/S0140-6736(11)60243-2

4. Heidari B. Knee osteoarthritis prevalence, risk factors, pathogenesis and features: part I. Caspian J Intern Med. 2011;2(2):205-212.

5. Palazzo C, Nguyen C, Lefevre-Colau MM, Rannou F, Poiraudeau S. Risk factors and burden of osteoarthritis. Ann Phys Rehabil Med. 2016;59(3):134-138. doi:10.1016/j.rehab.2016.01.006

6. O'Neill TW, McCabe PS, McBeth J. Update on the epidemiology, risk factors and disease outcomes of osteoarthritis. Best Pract Res Clin Rheumatol. 2018;32(2):312-326. doi:10.1016/j.berh.2018.10.007

7. Damen J, van Rijn RM, Emans PJ, et al. Prevalence and development of hip and knee osteoarthritis according to American College of Rheumatology criteria in the check cohort. Arthritis Res Ther. 2019;21(1):4. doi:10.1186/s13075-018-1785-7.

8. Martel-Pelletier J, Barr AJ, Cicuttini FM, et al. Osteoarthritis. Nat Rev Dis Primers. 2016;2:16072. doi:10.1038/nrdp.2016.72

9. Bernad-Pineda M, de Las HJ, Garcés-Puentes MV. [Quality of life in patients with knee and hip osteoarthritis]. Rev Esp Cir Ortop Traumatol. 2014;58(5):283-289. doi:10.1016/j.recot.2014.04.005

10. Bhala N, Emberson J, Merhi A, et al. Vascular and upper gastrointestinal effects of non-steroidal anti-inflammatory drugs: meta-analyses of individual participant data from randomised trials. Lancet. 2013;382(9894):769-779.

11. Bensman A. Non-steroidal Anti-inflammatory Drugs (NSAIDs) systemic use: the risk of renal failure. Front Pediatr. 2019;7:517. doi:10.3389/fped.2019.00517

12. Fratianni A, Ribeiro DCR, Leung KE, Drepper M. Prevention of high digestive hemorrhage on NSAID. Rev Med Suisse. 2020;16 (680):268-271.

13. McAlindon TE, Bannuru RR, Sullivan MC, et al. OARSI guidelines for the non-surgical management of knee osteoarthritis. Osteoarthritis Cartilage. 2014;22(3):363-388. doi:10.1016/j.joca.2014.01.003.

14. Brosseau L, Taki J, Desjardins B, et al. The Ottawa panel clinical practice guidelines for the management of knee osteoarthritis. Part one: introduction, and mind-body exercise programs. Clin Rehabil. 2017;31(5):582-595. doi:10.1177/0269215517691083.

15. Cutolo M, Berenbaum F, Hochberg M, Punzi L, Reginster JY. Commentary on recent therapeutic guidelines for osteoarthritis. Semin Arthritis Rheum. 2015;44(6):611-617. doi:10.1016/j. semarthrit.2014.12.003

16. Nazari A, Moezy A, Nejati P, Mazaherinezhad A. Efficacy of high-intensity laser therapy in comparison with conventional physiotherapy and exercise therapy on pain and function of patients with knee osteoarthritis: a randomized controlled trial with 12-week follow up. Lasers Med Sci. 2019;34(3):505-516. doi:10.1007/s10103018-2624-4

17. Henriksen M, Hansen JB, Klokker L, Bliddal H, Christensen R. Comparable effects of exercise and analgesics for pain secondary to knee osteoarthritis: a meta-analysis of trials included in Cochrane systematic reviews. J Comp Eff Res. 2016;5(4):417-431. doi:10.2217/cer-2016-0007

18. Brosseau L, Taki J, Desjardins B, et al. The Ottawa panel clinical practice guidelines for the management of knee osteoarthritis. Part two: strengthening exercise programs. Clin Rehabil. 2017;31 (5):596-611. doi:10.1177/0269215517691084.

19. Wang J, Yang L, Li Q, et al. Construction of an adherence rating scale for exercise therapy for patients with knee osteoarthritis. $B M C$ Musculoskelet Disord. 2018;19(1):263. doi:10.1186/s12891-0182200-x.

20. Lin LL, Li YT, Tu JF, et al. Effectiveness and feasibility of acupuncture for knee osteoarthritis: a pilot randomized controlled trial. Clin Rehabil. 2018;32(12):1666-1675. doi:10.1177/026921551879 0632 . 
21. Qiu JQ, Liu SR, Lin QL, Li MJ, Zhuang JX, Wu GW. Acupuncture combined with cinesiotherapy cupping for knee osteoarthritis with qi stagnation and blood stasis syndrome: a randomized controlled trial. Zhongguo Zhen Jiu. 2019;39(5):462-466. doi:10.13703/j.02552930.2019.05.002

22. Liu SR, Qiu JQ, Zhang LY, Lin QL, Ye GP, Zhu DY. Warming acupuncture combined with moxibustion at Yongquan (KI 1) for knee osteoarthritis with kidney-marrow deficiency: a randomized controlled trial. Zhongguo Zhen Jiu. 2019;39(8):799-803. doi:10. 13703/j.0255-2930.2019.08.001

23. Ozsoy Z, Demir E. Correction to: which bariatric procedure is the most popular in the world? A bibliometric comparison. Obes Surg. 2018;28(8):2353. doi:10.1007/s11695-018-3202-3

24. Zhang L, Yuan H, Zhang L, Li J, Li H. Effect of acupuncture therapies combined with usual medical care on knee osteoarthritis. $J$ Tradit Chin Med. 2019;39(1):103-110.

25. Wang TQ, Li YT, Wang LQ. et al. Electroacupuncture versus manual acupuncture for knee osteoarthritis: a randomized controlled pilot trial. Acupunct Med. 2020;2020:975696531

26. Chen N, Wang J, Mucelli A, Zhang X, Wang C. Electro-acupuncture is beneficial for knee osteoarthritis: the evidence from meta-analysis of randomized controlled trials. Am J Chin Med. 2017;45 (5):965-985. doi:10.1142/S0192415X17500513

27. Chen C, SanJuan FI, Hou J. The structure and dynamics of cocitation clusters: a multiple-perspective cocitation analysis. J Am Soc Info Ence Technol. 2010;61:7.

28. Belter CW. Bibliometric indicators: opportunities and limits. $J$ Med Libr Assoc. 2015;103(4):219-221. doi:10.3163/1536-5050.103.4.014

29. Synnestvedt MB, Chen C, Holmes JH, CiteSpace II: Visualization and knowledge discovery in bibliographic databases. AMIA Annu Symp Proc. 2005;2005:724-728

30. Zhang Y, Li C, Ji X, Yun C, Wang M, Luo X. The knowledge domain and emerging trends in phytoremediation: a scientometric analysis with CiteSpace. Environ Sci Pollut Res Int. 2020;27 (13):15515-15536. doi:10.1007/s11356-020-07646-2

31. Liang C, Luo A, Zhong Z. Knowledge mapping of medication literacy study: a visualized analysis using CiteSpace. Sage Open Med. 2018;6:2105872199. doi:10.1177/2050312118800199

32. AJ V, EA V, G L, et al. Acupuncture for chronic pain: update of an individual patient data meta-analysis. J Pain. 2018;19(5):455-474. doi:10.1016/j.jpain.2017.11.005

33. Wu SY, Lin CH, Chang NJ, et al. Combined effect of laser acupuncture and electroacupuncture in knee osteoarthritis patients: a protocol for a randomized controlled trial. Medicine. 2020;99(12):e19541. doi:10.1097/MD.0000000000019541.

34. Zhang Q, Fang J, Chen L, et al. Different kinds of acupuncture treatments for knee osteoarthritis: a multicentre, randomized controlled. Trial Trials. 2020;21(1):264. doi:10.1186/s13063-0194034-8.

35. Lv ZT, Shen LL, Zhu B, et al. Effects of intensity of electroacupuncture on chronic pain in patients with knee osteoarthritis: a randomized controlled trial. Arthritis Res Ther. 2019;21(1):120. doi:10.1186/ s13075-019-1899-6.
36. Luo L, Liao M, Peng JX, et al. Comparison of the efficacy between conventional moxibustion and smoke-free moxibustion on knee osteoarthritis: a randomized controlled trial. Evid Based Complement Alternat Med. 2019;2019:1291947. doi:10.1155/2019/ 1291947

37. Woods B, Manca A, Weatherly H, et al. Cost-effectiveness of adjunct non-pharmacological interventions for osteoarthritis of the knee. PLoS One. 2017;12(3):e172749. doi:10.1371/journal.pone.0172749.

38. SL P, J B, S C, H M. Insufficient evidence to determine the impact of patient preferences on clinical outcomes in acupuncture trials: a systematic review. J Clin Epidemiol. 2013;66(3):308-318. doi:10.1016/j.jclinepi.2012.09.011

39. GJ M, P P, M D, et al. A systematic review of evidence for the effectiveness of practitioner-based complementary and alternative therapies in the management of rheumatic diseases: osteoarthritis. Rheumatology. 2012;51(12):2224-2233. doi:10.1093/rheumatology/kes200

40. CM W. Clinical research on acupuncture concepts and guidance on efficacy and effectiveness research. Chin J Integr Med. 2011;17 (3):166-172. doi:10.1007/s11655-011-0662-x

41. Luo X, Hou XS, Tian ZY, Meng X, Li SM, Bai P. Randomized controlled clinical trial of acupuncture treatment for knee osteoarthritis in the early stage. Zhen Ci Yan Jiu. 2019;44(3):211-215. doi:10.13702/j.1000-0607.180677

42. HP S, U M, K S, et al. Acupuncture and knee osteoarthritis: a three-armed randomized trial. Ann Intern Med. 2006;145(1):12-20. doi:10.7326/0003-4819-145-1-200607040-00005

43. Witt C, Brinkhaus B, Jena S, et al. Acupuncture in patients with osteoarthritis of the knee: a randomised trial. Lancet. 2005;366 (9480):136-143. doi:10.1016/S0140-6736(05)66871-7.

44. Hinman RS, McCrory P, Pirotta M, et al. Acupuncture for chronic knee pain: a randomized clinical trial. JAMA. 2014;312 (13):1313-1322. doi:10.1001/jama.2014.12660.

45. Mavrommatis CI, Argyra E, Vadalouka A, et al. Acupuncture as an adjunctive therapy to pharmacological treatment in patients with chronic pain due to osteoarthritis of the knee: a 3-armed, randomized, placebo-controlled trial. Pain. 2012;153(8):1720-1726.

46. Zheng K, Wang X. Publications on the association between cognitive function and pain from 2000 to 2018: a bibliometric analysis using CiteSpace. Med Sci Monit. 2019;25:8940-8951. doi:10.12659/ MSM.917742

47. D M, S H, KF S, et al. CONSORT 2010 explanation and elaboration: updated guidelines for reporting parallel group randomised trials. Int J Surgery. 2012;10(1):28-55. doi:10.1016/j.ijsu.2011.10.001

48. EJ K, CY L, EY L, SD L, KS K. Comparing the effects of individualized, standard, sham and no acupuncture in the treatment of knee osteoarthritis: a multicenter randomized controlled trial. Trials. 2013;14:129. doi:10.1186/1745-6215-14-129

49. JY Z, L L, LL Z, et al. Moxibustion versus diclofenac sodium gel for the treatment of knee osteoarthritis: a study protocol for a double-blinded, double-placebo, randomised controlled trial. $B M J$ Open. 2017;7(4):e12879.

50. A M. The future of osteoarthritis therapeutics: targeted pharmacological therapy. Curr Rheumatol Rep. 2013;15(10):364. doi:10.1007/ s11926-013-0364-9 


\section{Publish your work in this journal}

The Journal of Pain Research is an international, peer reviewed, open access, online journal that welcomes laboratory and clinical findings in the fields of pain research and the prevention and management of pain. Original research, reviews, symposium reports, hypothesis formation and commentaries are all considered for publication. The manuscript management system is completely online and includes a very quick and fair peer-review system, which is all easy to use. Visit http:// www.dovepress.com/testimonials.php to read real quotes from published authors. 